\title{
Use of Resin Solvent as a Facilitator for Removal of Resin Composite Restoratives by Influencing their Mechanical Properties: Is this Possible?
}

\author{
Inas A Elghandour
}

\begin{abstract}
Aim: The aim is to evaluate the influence of resin solvent on Vickers microhardness, flexural strength, and flexural modulus of a resin composite in order to test the ability of resin solvent to soften or weaken resin composite during replacement of failed restoration.

Materials and methods: Fifty specimens were prepared from Tetric N-Ceram; forty used for flexural strength and elastic modulus tests and ten used for microhardness test. For flexural strength; they were divided into four groups of ten specimens each; in first group; specimens were not subjected to resin solvent (control), in second, third and fourth groups; specimens were subjected to resin solvent for 1,3 , and 5 minutes respectively. For microhardness, specimens were examined before after application of resin solvent for 1, 3, and 5 minutes with a total of forty readings. Collected data were statistically analyzed.

Results: Resin solvent was found to reduce significantly the flexure strength of Tetric N-Ceram compared to the control group. Differences in flexure strength between 1,3 , and 5 minutes applications were statistically nonsignificant. For elastic modulus and microhardness, the influence of resin solvent on resin composite was statistically nonsignificant.

Conclusion: Resin solvent weakens the flexural strength of resin composite but did not affect its microhardness or elastic modulus. Increasing the time of application from 1, 3, and 5 minutes did not further reduce the flexural strength.

Clinical significance: Resin solvent had shown promising potentials to reduce the strength and hence facilitate removal of failed composite restoration during replacement.
\end{abstract}

Keywords: Laboratory research, Mechanical properties, Removal of resin composite, Resin composites, Resin remover, Resin solvent. Journal of Operative Dentistry and Endodontics (2019): 10.5005/jp-journals-10047-0074

\section{INTRODUCTION}

Resin composite restoratives are essential materials that are used nowadays for esthetic replacement of defective hard tooth structure. It has esthetic qualities that approach that of natural teeth. However, it suffers polymerization shrinkage and hence micro leakage that could lead to postoperative sensitivity, discoloration or recurrent caries with subsequent failure of the restoration. ${ }^{1}$ These defects might require repair or replacement of the restoration. In addition, after the removal of braces following orthodontic treatment, remnants of resin composite require removal with no loss of hard tooth structure. The tooth/resin composite boundaries might be difficult to discern, which might lead to removal of unaffected tooth structure. An agent that could change the texture or properties of resin composites in order to facilitate its removal is required. ${ }^{2}$

Most operators use visual inspection and tactile sensation to differentiate resin composites from tooth structure depending solely on their clinical experience and skills. With improvements in resin composites nowadays, this visual inspection might be difficult during removal of previous composite restoration. ${ }^{3}$ Previous researchers had shown that softening of resin composites is possible using chemical agents as ethanol, heptane, lactic acid, and cyclohexanone. ${ }^{4}$ However, to establish softening of resin composite by these materials, the restoration had to be soaked in them for long period, 24 hours which is not feasible in a dental clinic to establish softening for removal of restoration. ${ }^{5}$ In addition, it was shown that storage of resin composite in some of these materials
Department of Restorative Dental Science, College of Dentistry, Imam Abdul Rahman Bin Faisal University, Dammam, Kingdom of Saudi Arabia; Department of Operative Dentistry, Faculty of Dentistry, Cairo University, Cairo, Egypt

Corresponding Author: Inas A Elghandour, Department of Restorative Dental Science, College of Dentistry, Imam Abdul Rahman Bin Faisal University, Dammam, Kingdom of Saudi Arabia; Department of Operative Dentistry, Faculty of Dentistry, Cairo University, Cairo, Egypt, Phone: +20 1145195315, e-mail: inaselghan@cu.edu.eg

How to cite this article: Elghandour IA. Use of Resin Solvent as a Facilitator for Removal of Resin Composite Restoratives by Influencing their Mechanical Properties: Is this Possible? J Oper Dent Endod 2019;4(1):1-5.

Source of support: Nil

Conflict of interest: None

heptane and $2 \%$ citric acid did not affect flexure strength of resin composites even after storage for one week. ${ }^{6}$

During endodontic treatment, resin-based filling materials revealed better bonding to dentin with less microleakage compared gutta-percha endodontic filling. ${ }^{7}$ Resin solvent materials have been introduced to facilitate the removal of resin-based endodontic fillings during endodontic retreatment. ${ }^{8}$ Chloroform, xylene, halothane, eucalyptol, and orange oil have been used to remove gutta-percha. ${ }^{9}$ EndoSolv $\mathrm{R}$ was designated to remove phenol-based resin sealers. It was reported that the usage of Endosolv $\mathrm{R}$ solvent could reduce working time during the removal of previous endodontic filling. ${ }^{10}$ 
Shenoi et al. ${ }^{11}$ found that usage of Endosolv R for 2 minutes softened resin-based root canal sealers. Mushtaq et al. ${ }^{12}$ found that xylene, after application for 2 minutes, dissolved resilon filling more than refined orange oil and tetrachloroethylene in descending order. On increasing immersion time to 5 minutes, the dissolving capacity of solvents was increased except for xylene solvent.

So, a question arises; would resin solvents, used for softening of resin-based root canal obturating materials, be effective in softening or weakening of failed resin composite restoration, hence facilitating its removal? In addition, would the time of immersion inside a resin solvent affect the mechanical properties of resin composite in order to enhance its removal? Accordingly, it was hypothesized that resin solvent could reduce flexural strength, flexural modulus, or microhardness of resin composite in order to facilitate removal of resin composite restoration in case of failure. The second hypothesis was that increasing time of exposure to resin solvent would enhance the reduction of strength, modulus, or surface microhardness.

\section{Materials and Methods}

\section{Materials}

Tetric N-Ceram nanocomposite was used in the present study (Ivoclar VivadentTM, Schaan, Liechtenstein). It is composed of barium glass, prepolymerized filler ( $56 \%$ vol.), mixed oxide, ytterbium trifluoride, dimethacrylate, catalysts, stabilizers, additives, and pigments. A universal shade A3 was selected.

The resin solvent; resin remover brand (Produits Dentaires SA, 1800 Vevey, Switzerland) is composed of oil of citronella (15\%), $\mathrm{N}, \mathrm{N}$-dimethylformamide (5\%), tolu balsam (5\%), tincture of benzoin (5\%), essential oils (4.6\%) excipient ad (100\%). According to the manufacturer, it is used for softening of resin-based root canal filling materials.

\section{Flexural Strength and Flexural Modulus}

Forty bar-shaped specimens were fabricated following ISO 4049 specifications and manufacturer's instructions. ${ }^{13}$ Composite was applied inside a prefabricated Teflon mold $(25 \mathrm{~mm}$ length $\times 2 \mathrm{~mm}$ width $\times 2 \mathrm{~mm}$ height). A Mylar strip was pressed over the specimen's surface, and excess material was removed. The specimen was cured for 40 seconds using a light-emitting diode curing unit (LEDition Ivoclar VivadentTM, Schaan, Liechtenstein). The curing unit had a light intensity of $600 \mathrm{~mW} / \mathrm{cm}^{2}$ and a wavelength range of $430-490 \mathrm{~nm}$. To ensure proper curing of resin composite, an overlapping technique was used during curing. Finishing was done using Soflex discs. Specimens were stored in water for 1-week at room temperature. Specimens were divided into four groups, of ten specimens each, according to the treatment received. In the first group, no treatment was performed using resin solvent (control group). In the other three groups, specimens were soaked in resin solvent for 1, 3, and 5 minutes respectively. Soaking was made in order to simulate the removal of complex restorations where resin composite is exposed to solvent from more than one surface. After soaking, specimens were rinsed using water and tested for flexure strength using a universal testing machine at a speed of $0.5 \mathrm{~mm} /$ minute (Instron 8871; Instron Co., Norwood, MA, USA). The maximum fracture load $(F$, in $N)$ of each specimen was recorded, and the flexural strength $O_{f}^{\prime}$, in $\mathrm{MPa}$, was calculated by the equation

$$
O_{f}^{\prime}=\frac{3 P L}{2 b h^{2}}
$$

where $O_{f}^{\prime}$ is the flexure stress, $L$ is the distance between the supporting rollers, $b$ represents the specimen width, and $h$ represents the specimen height.

The flexure modulus of the specimens subjected to flexure strength testing was measured using the same universal testing machine (Instron 8871; Instron Co., Norwood, MA, USA). It was calculated using the form ula

$$
E_{f}=\frac{L^{3}(P)}{48 l \Delta}
$$

where $E_{f}$ represents modulus of elasticity calculated during flexural strength testing, $p / \Delta$ is the slope for load deflection, and $I$ is the moment of inertia

\section{Vickers Microhardness}

Ten disk-shaped specimens were prepared in a plastic mold of dimensions $5 \mathrm{~mm}$ diameter $\times 2 \mathrm{~mm}$ height. Tetric $\mathrm{N}$-Ceram composite was packed in one increment inside the mold. The surface of each specimen was covered by Mylar strip and pressed using a glass slab to extrude excess material. Curing was performed following the manufacturer's instructions using LEDition curing unit (Ivoclar VivadentTM, Schaan, Liechtenstein) for 40 seconds. The tip of curing unit was placed in contact with the surface of the sample during curing. Samples were polished using Soflex discs (3M ESPE, St. Paul, MN, USA) under air-water spray. They were stored in water for 1-week at room temperature. The surface microhardness was measured using Vickers microhardness tester (Micromet 6049 Microhardness Testing Machine, Buehler, Illinois, USA). Three readings were taken from each specimen, and the mean was calculated. For randomization, specimens were casually turned before indentations. A load of $200 \mathrm{~g}$ was applied for 15 seconds during each reading. Specimens were measured for surface microhardness before treatment with resin solvent (control group) and after 1, 3, and 5 minutes treatment with the resin solvent.

\section{Statistical Analysis}

Statistical analysis was performed with $\mathrm{IBM}^{\circledR}{ }^{\circledR}$ SPSS ${ }^{\circledR}$ Statistics Version 22 for Windows. Quantitative data were shown as the mean, standard deviation (SD), median, range (minimum-maximum), and $95 \%$ confidence interval $(95 \% \mathrm{Cl})$ for the mean values (Table 1). Data distribution was checked, and then Kolmogorov-Smirnov and Shapiro-Wilk tests were used to explore the normality of data. Flexural strength and microhardness data showed normal (parametric) distribution. Flexural modulus showed non-normal (nonparametric) distribution. For parametric data, a one-way ANOVA test was used to compare the four groups followed by Bonferroni pairwise test. Kruskal-Wallis test was used to compare the four groups for nonparametric data. Microhardness comparisons groups and overtime were done by mixed model ANOVA for the repeated measure. The significance level was set at $p \leq 0.05$.

\section{Results}

\section{Flexural Strength and Flexural Modulus}

The mean flexure strength (SD) for the control group was $48.0 \pm$ 23.3. For other groups, it was $30.5 \pm 6.2,28.4 \pm 14.2$ and $26.9 \pm 5.7$ for 1 minute, 3 minutes, and 5 minutes respectively, as shown in Table 1. This was statistically significant at $p$ value $=0.007$. Pairwise comparisons revealed that the control group was statistically significant, from all other groups; other groups comparisons were not statistically significant. 


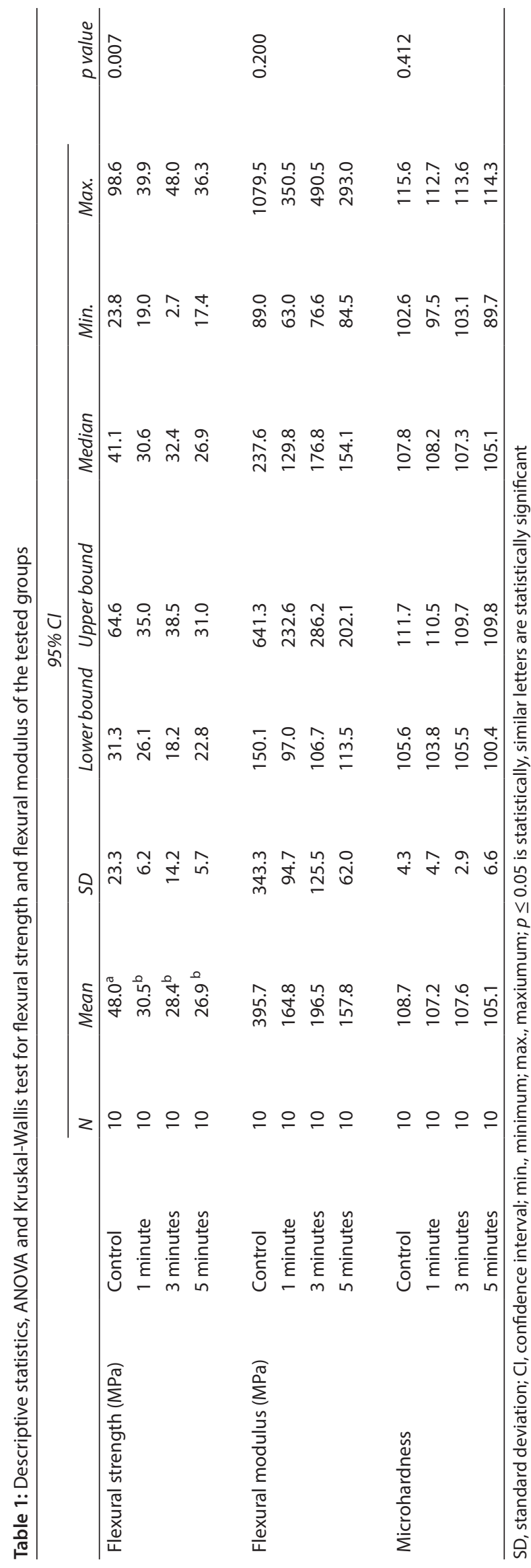

The mean flexural modulus (SD) for different groups was $395.7 \pm$ $343.3,164.8 \pm 94.7,196.5 \pm 125.5,157.8 \pm 62.0$ for the control group, 1 minute, 3 minutes, and 5 minutes groups, respectively (Table 1). The differences were not statistically significant at $p$ value $=0.2$.

\section{Vickers Microhardness (Fig. 1)}

The mean microhardness strength (SD) for different groups was $108.7 \pm 4.3,107.2 \pm 4.7,107.6 \pm 2.9,105.1 \pm 6.6$ for control group, 1 minute, 3 minutes, and 5 minutes groups, respectively (Table 1). The differences were not statistically significant at $p$ value $=0.412$.

\section{Discussion}

Flexural strength denotes the ability of the material to bend before breakage. ${ }^{14}$ Hardness test evaluates the degree of polymerization of resin composites. ${ }^{15}$ In the present study, repeated hardness measurements were made before and after immersion of resin composite in resin solvent in order to evaluate the degree of softening. ${ }^{16}$ The softening effect of any agent on resin composite is determined by the degree of matching of the solubility parameter of the solvent to that of the resin matrix. ${ }^{17}$ Solubility parameters of BISGMA are (21.5 MJ1/2/m3/2 as determined by Asmussen and Uno. ${ }^{18}$ Solubility parameter of $75 \%$ ethanol is $31 \cdot 5$, acetic acid $20 \cdot 7$, acetone $20 \cdot 3$, polyacrylic acid $18 \cdot 0 .{ }^{19}$ No data about the solubility parameter of resin remover components and its matching to resin composites were found which requires further research. In the present study, the time used for application of resin solvent is few minutes as this time is feasible clinically and follows the recommendation of usage of resin remover by the manufacturer.

In the present study, it was found that the resin solvent reduced the flexure strength of Tetric $\mathrm{N}$-Ceram significantly compared to control. However, the effect of time of application of resin solvent from 1-minute to 5 minutes did not produce a statistically significant effect on its flexure strength. This reduction in strength might be due to deterioration of resin components of the resin matrix by the resin solvent. The presence of prepolymerized fillers in the material might also enhance the influence of resin solvent, considering the resin content of fillers. No previous work has been found regarding the effect of resin solvent on flexure strength of resin composites. However, other researchers studied the effect of other solvents. Randolph et al. ${ }^{20}$ found that most prepolymerized fillers containing resin composites, as one used in the present study, displayed relatively low flexure modulus and strength considering their high filler content. They mentioned that the fillers should have an elastic modulus higher than the organic phase to be effective in reinforcement of resin matrix. They also found that flexure strength was correlated with sorption, where increased sorption was associated with decreased strength. Gundogdu et al. ${ }^{21}$ found that storage of resin composites in mouthwash for 24 hours and 7 days, did not affect significantly their flexure strength. This contradicts the finding of present study. However, the materials, time, and solvents used were different between the two studies.

For modulus of elasticity and surface microhardness, the resin solvent did not produce any statistically significant changes of resin composite regardless of application time. This implies that the resin solvent used had limited time to soften the surface, but it has enough time to enter inside the material and affect its flexural strength. Surface microhardness of composite resin is correlated with the volume, size, and nature of filler. ${ }^{22}$ Consequently, this property would not be affected if microhardness' indentation had the same dimensions as fillers. Regarding the matrix type, the resin 

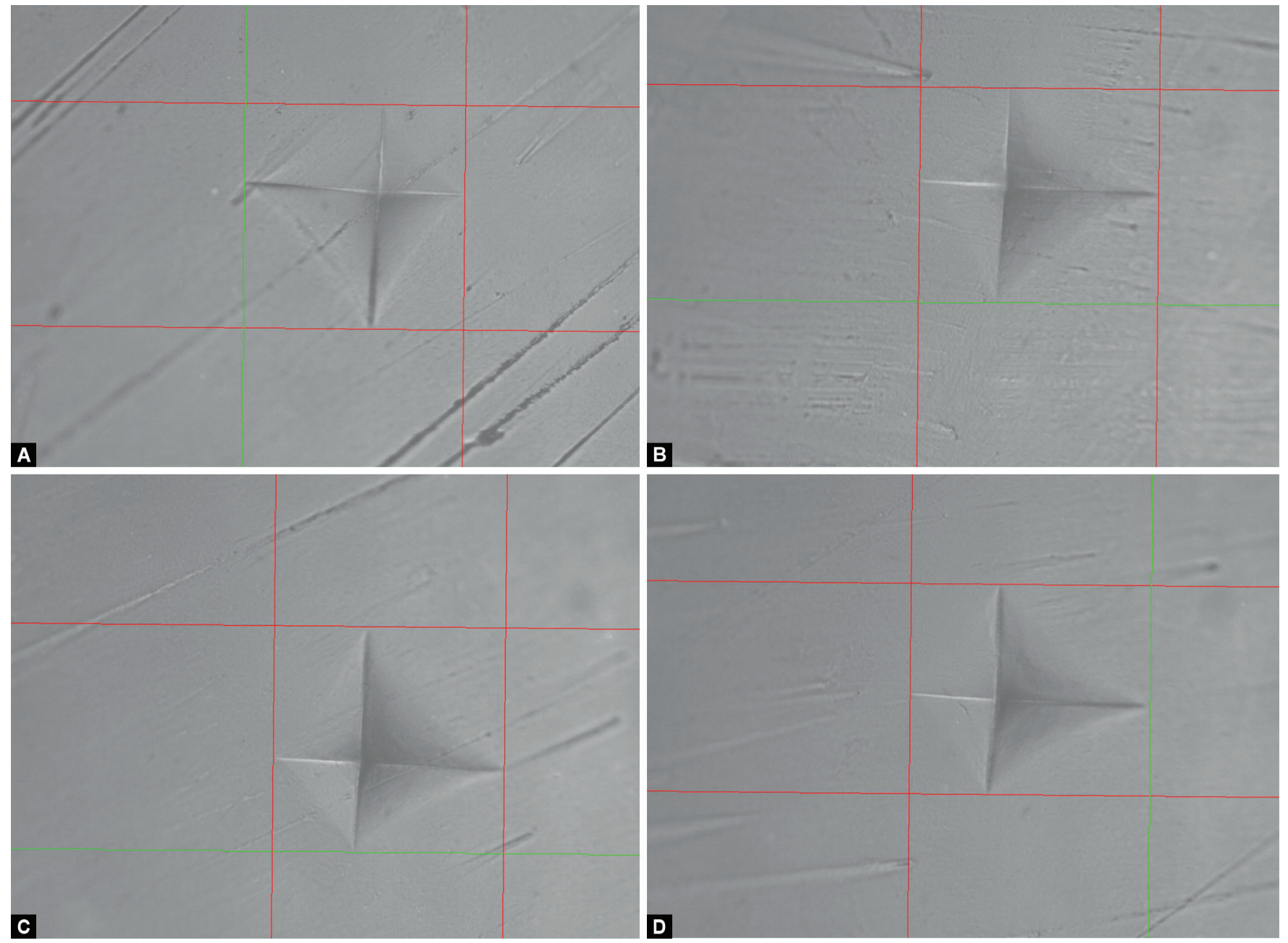

Figs 1 A to D: Photomicrographs of Tetric N Ceram (A) Before application of resin solvent; (B) After application of resin solvent for 1 minute; (C) 3 minutes; (D) 5 minutes

matrix of Tetric $\mathrm{N}$-Ceram is dimethacrylate based resin. Soderholm et al. ${ }^{23}$ found that UDMA based matrix showed higher wear resistance when compared to Bis-GMA based materials. No previous research has been found considering the effect of resin solvent on microhardness of resin composite. Some researchers reported that there is no influence of acidic solutions used for a prolonged period on the degradation of resin-based composites. ${ }^{24,25}$ However, other studies have reported that acidic beverages could influence the physical properties of resin composites. ${ }^{26,27}$ Shenoi et al. ${ }^{11}$ found that, after 2 minutes application, Endosolv R produced significant softening of $\mathrm{AH} 26$, AH Plus, Adseal epoxy resin-based sealers. The softening effect of xylene to epoxy resin sealers was brand dependent where xylene softened AH Plus and Adseal more than $\mathrm{AH} 26$. Increasing time from 1 to 2 minutes increased the softening effect for both Endosolv $\mathrm{R}$ and xylene solvents. This contradicted the findings of present research regarding effect of time. However, the materials and solvent tested were different in both studies. Cruickshank and Chadwick ${ }^{19}$ investigated the softening effect of (polyacrylic acid, 75\% ethanol, acetic acid, and acetone), applied for 3 minutes, on anterior resin composites (Blend-a-Lux, Prisma $\mathrm{APH}$, and Brilliant). They studied the relationship between usage of softening agent and extent of cavity enlargement done during removal of resin composite restoration. They found that reduction of microhardness of resin composites was brand dependent where acetic acid reduced microhardness of Blend-a-Lux and $75 \%$ ethanol reduced microhardness of Prisma-APH. However, this reduction did not reduce the cavity enlargement during removal of restoration. So further studies with other brands of resin composite are required to study the softening effect of resin solvent.

In the present study, the resin solvent reduced the flexural strength but did not influence greatly the elastic modulus and microhardness of tested resin composite. This indicated that flexural strength was influenced by the strength of resin matrix more than microhardness that is more affected by filler content. The resin solvent is aimed to break down resin matrix. So, deterioration of flexural strength was evident. The time of application is limited to affect the microhardness intensively. This was in accordance with Sabbagh et al. ${ }^{28}$ who compared the hardness and flexural strength of expired versus non-expired resin composites. They found that the flexural strength was reduced for expired composites compared to non-expired ones. Meanwhile, expiration date did not affect the microhardness of specimens with no correlation to the reduction of flexural strength. Randolph et al. ${ }^{20}$ found that microhardness and flexural strength were reduced in some brands of resin composite by storage in ethanol. Meanwhile, in other brands, flexural strength was reduced while microhardness was not affected. This indicates that reduction of both or either of these properties is brand dependent. Lima et al. ${ }^{29}$ found that the Knoop hardness values were 
statistically higher at top surface $(0.5 \mathrm{~mm})$ compared to interior of resin composite $(4 \mathrm{~mm})$ for Tetric $\mathrm{N}$-Ceram Bulk-Fill. However, the biaxial flexural strength did not differ significantly between the top and $4 \mathrm{~mm}$ depth for resin composite.

During removal of a failed resin composite restoration, the material is already exposed to several materials- oral environment interactions, which might enhance its degradation by resin solvent. This research is an in vitro assay; therefore, the results should be carefully interpreted, as the specimens were not exposed to environmental degradation. The inclusion of only one brand of resin composites requires further research on other materials and clinical studies should be conducted to confirm the results. Also, application of resin solvent might affect the bonding efficacy of resin composite applied later on, which demands further research.

For the first hypothesis, it was partially accepted, where the resin solvent was able to reduce the flexure strength but did not affect significantly the surface microhardness or flexural modulus. For the second hypothesis, it was rejected, as time did not affect flexural strength, flexural modulus, or surface microhardness.

\section{ConCLUSION}

Resin solvent showed the ability to reduce the flexure strength of Tetric N-Ceram compared to the control group. The differences between 1, 3, and 5 minutes was nonsignificant. On the contrary, resin solvent showed a nonsignificant influence on elastic modulus and microhardness of resin composite.

\section{References}

1. Villalta $P, L u H$, et al. Effects of staining and bleaching on color change of dental composite resins. J Prosthet Dent 2006;95:137-142. DOI: 10.1016/j.prosdent.2005.11.019.

2. Abdallah MN, Light $N$, et al. Development of a composite resin disclosing agent based on the understanding of tooth staining mechanisms. J Dent 2014;42:697-708. DOI: 10.1016/ j.jdent.2014.03.004.

3. Meller C, Klein C. Fluorescence properties of commercial composite resin restorative materials in dentistry. Dent Mater 2012;31:916-923. DOI: 10.4012/dmj.2012-079.

4. Chadwick RG. Thermocycling - the effects upon the compressive strength and abrasion resistance of three resin composites. J Oral Rehabil 1994;21:533-543. DOI: 10.1111/j.1365-2842.1994.tb01167.x.

5. Inagaki LT, Dainezi VB, et al. Evaluation of sorption/solubility, softening, flexural strength and elastic modulus of experimental resin blends with chlorhexidine. J Dent 2016;49:40-45. DOI: 10.1016/ j.jdent.2016.04.006.

6. Mohammadi E, Pishevar $L$, et al. Effect of food simulating liquids on the flexural strength of a methacrylate and silorane-based composite. PLoS One 2017;12:e0188829. DOI: 10.1371/journal.pone.0188829.

7. Punia SK, Nadig P, et al. An in vitro assessment of apical microleakage in root canals obturated with gutta-flow, resilon, thermafil and lateral condensation: a stereomicroscopic study. J Conserv Dent 2011;14:173-177. DOI: 10.4103/0972-0707.82629.

8. Martos J, Gastal MT, et al. Dissolving efficacy of organic solvents on root canal sealers. Clin Oral Invest 2006;10:50-54. DOI: 10.1007/ s00784-005-0023-2.

9. Johann JE, Martos J, et al. Use of organic solvents in endodontics: a review. Clin Pesq Odontol Curitiba 2006;2:393-399.

10. Saglam BC, Kocak MM, et al. Efficacy of different solvents in removing gutta-percha from curved root canals: A micro-computed tomography study. Aust Endod J 2014;40:76-80. DOI: 10.1111/ aej.12041.

11. Shenoi PR, Badole GP, et al. Evaluation of softening ability of Xylene \& Endosolv-R on three different epoxy resin based sealers within 1 to 2 minutes-an in vitro study. Restor Dent Endod 2014;39: 17-23. DOI: 10.5395/rde.2014.39.1.17.

12. Mushtaq M, Farooq R, et al. Dissolving efficacy of different organic solvents on gutta-percha and resilon root canal obturating materials at different immersion time intervals. J Conserv Dent 2012;15:141-145. DOI: 10.4103/0972-0707.94584.

13. International Standards Organization. ISO 4049 Dentistry - Polymerbased filling, restorative and luting materials; 2009.

14. Meenakumari C, Bhat KM, et al. Evaluation of mechanical properties of newer nanoposterior restorative resin composites: An In vitro study. Contemp Clin Dent 2018;9:S142-S146. DOI: 10.4103/ccd.ccd_160_18.

15. Ilie N, Hilton TJ, et al. Academy of Dental Materials guidance-Resin composites: Part I-Mechanical properties. Dent Mater 2017;33: 880-894. DOI: 10.1016/j.dental.2017.04.013.

16. Feitosa VP, Fugolin APP, et al. Effects of different photo-polymerization protocols on resin-dentine mTBS, mechanical properties and crosslink density of a nano-filled resin composite. J Dent 2012;40:802-809. DOI: 10.1016/j.jdent.2012.05.014.

17. Lee SY, Greener EH, et al. Effect of food and oral simulating fluids on structure of adhesive composite systems. J Dent 1995;23:27-35. DOI: 10.1016/0300-5712(95)90657-4.

18. Asmussen E, Uno S. Solubility Parameters, Fractional Polarities, and Bond Strengths of Some Intermediary Resins Used in Dentin Bonding. J Dent Res 1993;72:558-565. DOI: 10.1177/00220345930720030101.

19. Cruickshank EJ, Chadwick RG. Can chemical softening agents minimize cavity enlargement during removal of failed anterior resin composite restorations? J Oral Rehabil 1998;25:167-173. DOI: 10.1046/j.1365-2842.1998.00226.x.

20. Randolph LD, Palin WM, et al. Filler characteristics of modern dental resin composites and their influence on physico-mechanical properties. Dent Mater 2016;32:1586-1599. DOI: 10.1016/j.dental. 2016.09.034.

21. Gundogdu M, Kurklu D, et al. The Evaluation of Flexural Strength of Composite Resin Materials with and without Fiber. Dent 2014;4: 259-265. DOI: 10.4172/2161-1122.1000259.

22. Oberholzer TG, Grobler SR, et al. The effects of light intensity and method of exposure on the hardness of four light-cured dental restorative materials. Int Dent J 2003;53:211-215. DOI: 10.1111/j.1875595X.2003.tb00747.x.

23. Soderholm KJ, Lambrechts $P$, et al. Clinical wear performance of eight experimental dental composites over three years determined by two measuring methods. Eur J Oral Sci 2001;109:273-281. DOI: 10.1034/j.1600-0722.2001.00064.x.

24. Patel SB, Gordan VV, et al. The effect of surface finishing and storage solutions on the color stability of resin-based composites. J Am Dental Assoc 2004;135:587-594. DOI: 10.14219/jada.archive.2004.0246.

25. Moon JD, Seon EM, et al. Effect of immersion into solutions at various $\mathrm{pH}$ on the color stability of composite resins with different shades. Restor Dent Endod 2015;40:270-276. DOI: 10.5395/rde.2015.40.4.270.

26. Xavier AM, Sunny SM, et al. Repeated exposure of acidic beverages on esthetic restorative materials: an in-vitro surface microhardness study. J Clin Exp Dent 2016;8:e312-e317. DOI: 10.4317/jced.52906.

27. Rajavardhan K, Sankar A, et al. Erosive potential of cola and orange fruit juice on tooth colored restorative materials. Ann Med Health Sci Res 2014;4:S208-S212. DOI: 10.4103/2141-9248.141960.

28. Sabbagh J, Nabbout F, et al. The effect of expiration date on mechanical properties of resin composites. J Int Soc Prevent Communit Dent 2018;8:99-103. DOI: 10.4103/jispcd.JISPCD_445_17.

29. Lima RBW, Gómez FM, et al. Bulk-fill resin composites: Mechanical properties vs depth of cure. 2016;32:e20-e21. 